Published in final edited form as:

Shock. 2008 January ; 29(1): 104-111. doi:10.1097/SHK.0b013e318067da56.

\title{
A MATHEMATICAL SIMULATION OF THE INFLAMMATORY RESPONSE TO ANTHRAX INFECTION
}

\author{
Rukmini Kumar ${ }^{*}$, Carson C. Chow ${ }^{\dagger}$, John D. Bartels ${ }^{\ddagger}$, Gilles Clermont ${ }^{\S}$, and Yoram \\ Vodovotz" \\ "Department of Physics, University of Pittsburgh \\ tDepartment of Mathematics, University of Pittsburgh \\ \#Immunetrics, Inc \\ §Department of Critical Care Medicine, University of Pittsburgh, Pittsburgh, Pennsylvania \\ "Department of Surgery, University of Pittsburgh, Pittsburgh, Pennsylvania
}

\begin{abstract}
Bacillus anthracis (anthrax) can trigger an acute inflammatory response that results in multisystem organ failure and death. Previously, we developed a mathematical model of acute inflammation after gram-negative infection that had been matched qualitatively to literature data. We modified the properties of the invading bacteria in that model to those specific to $B$. anthracis and simulated the host response to anthrax infection. We simulated treatment strategies against anthrax in a genetically diverse population including the following: (1) antibiotic treatment initiated at various time points, (2) antiprotective antigen vaccine, and (3) a combination of antibiotics and vaccine. In agreement with studies in mice, our simulations showed that antibiotics only improve survival if administered early in the course of anthrax infection. Vaccination that leads to the formation of antibodies to protective antigen is anti-inflammatory and beneficial in averting shock and improving survival. However, antibodies to protective antigen alone are predicted not to be universally protective against anthrax infection. Rather, our simulations suggest that an optimal strategy would require both vaccination and antibiotic administration.
\end{abstract}

\section{Keywords}

Anthrax; vaccine; biowarfare; mathematical model; simulation; sepsis

\section{INTRODUCTION}

The history of human infection with anthrax is centuries old. More recently, much has been learned about the molecular pathology of this bacterium, including the sequencing of Bacillus anthracis $(1,2)$. These developments have been spurred on by the potential and, more recently, actual use of this bacterium as a weapon of bioterror $(3,4)$. Anthrax modulates the acute inflammatory response of the host, and systemic anthrax infection is thought to result in a condition similar to sepsis and septic shock.

Copyright $\odot 2007$ by the Shock Society

Address reprint requests to Gilles Clermont, Department of Critical Care Medicine, University of Pittsburgh, 606B Scaife Hall, 3550 Terrace St, Pittsburgh, PA 15261. cler@ pitt.edu.

On-line supplementary material accompanies this article at www.shockjournal.com. 
Three basic routes of infection are known for anthrax: cutaneous, gastrointestinal, and inhalational. Inhalational anthrax is the most lethal form of the disease and the most likely route to be used as a bioterror weapon (5). After a variable incubation period, clinical symptoms are those caused by a significant systemic inflammatory response: fever, coughing, myalgia, and malaise. The disease becomes much more severe after 1 to 3 days, leading to dyspnea, strident cough, chills, and eventually death from multiorgan failure (6, 7). Therapeutic approaches for inhalational anthrax are no less complex than those for sepsis and are limited in their effectiveness (8). The current strategy is to provide organ support and administer high doses of antibiotics. This strategy is only efficacious if given sufficiently early in the course of the disease. There is therefore a great emphasis on developing effective and safe human vaccines against anthrax infection (9-11). However, the dangerous nature of anthrax makes human testing of vaccines impractical (12). Thus, it is not fully known how effective vaccines will be and how they should be best used.

Mathematical modeling may provide insight and predictions as to the effectiveness of proposed therapies against anthrax infection, using data on the time course of anthraxinduced inflammation. We previously published a general model of sepsis in response to infection and endotoxin administration (13) and modified it to account, in a quantitative fashion, for inflammation induced by diverse causes (14). Here, we report on the adaptation of this model to the specific case of inhalational anthrax, incorporating spore germination, the synthesis and relevant actions of lethal toxin and edema toxin, and other specific features of anthrax on the immune response. We used this model to simulate the biological response to anthrax infection and generate predictions for various treatment regimens that include vaccination and antibiotics. As an extension of our previous work on gram-negative sepsis (13), we carried out an in silico clinical trial to address population diversity in the response to such combination therapy.

\section{MATERIALS AND METHODS}

\section{Simulating anthrax infection}

Pathogenesis of anthrax infection-Several discrete events occur after a localized bacterial infection, which the host detects either directly or through the recognition of pathogen-derived immunostimulants such as gram-negative endotoxin (lipopolysaccharide [LPS]) or gram-positive lipoteichoic acid via a system of pattern recognition receptors (15). These events involve immune cells such as macrophages andneutrophils, proinflammatory andanti-inflammatory cytokinessuch asTNF, IL-1, IL-6, IL-10, and transforming growth factor (TGF) $\beta 1$. Proinflammatory cytokines also induce macrophages and neutrophils to produce free radicals (e.g., $\mathrm{NO}$, superoxide $\left[\mathrm{O}_{2}^{-}\right]$, hydroxyl radical $[\mathrm{OH}]$, and hydrogen peroxide $\left[\mathrm{H}_{2} \mathrm{O}_{2}\right]$ ) and degradative proteins (e.g., myeloperoxidase, bactericidal permeability increasing protein) that are directly toxic to bacteria and tissue. With the exception of NO, which may also help to protect the body's cells from damage/dysfunction during sepsis, these molecules are also toxic to the body's own cells, and the induced tissue damage or dysfunction can incite more inflammation (16).

Ideally, the immune response eliminates the pathogen, initiates appropriate repair mechanisms, and then subsides. In some cases, the response is not sufficiently vigorous to clear the pathogen. In other cases, pathogen is cleared, but positive feedback among components of the inflammatory response may lead to severe nonabating inflammation, manifested as shock, organ failure, and death (17). Although this is a simplification of the pathogenesis, the idea is supported by the presence of high levels of circulating proinflammatory cytokines in shock, their prognostic value in clinical sepsis, and the efficacy of their manipulation in animal models of sepsis $(18,19)$. 
The pathogenesis of anthrax involves toxins absent in standard gram-negative bacterial infections, and death from inhalational anthrax is thought to occur through the common process of systemic inflammation and bacteremia $(5,20)$. As depicted schematically in Figure 1, we consider the infection to be initiated by inhalation of aerosolized anthrax spores, which are internalized by monocytes/ macrophages/dendritic cells. The spores can incubate for a period of over 40 days (modal incubation period, $0-10$ days) during which time monocytes and immature dendritic cells may circulate and therefore spread latent anthrax infection throughout the body. Given the appropriate temperature and/or $\mathrm{CO}_{2}$ levels, the spores germinate (21).

The major toxins of $B$. anthracis are protective antigen, lethal factor, and edema factor. These three exotoxins are synthesized, and each forms heterodimers that result in bioactive molecules: edema toxin is a heterodimer of protective antigen and edema factor, and lethal toxin consists of protective antigen bound to lethal factor (5). Edema factor is a calmodulindependent adenylate cyclase, which causes elevations of the intracellular second messenger cyclic adenosine monophosphate. Edema toxin attracts neutrophils to the site of infection but suppresses the capacity of these cells to phagocytose bacteria and to produce superoxide in response to phagocytosis (22). Lethal factor, a zinc metalloprotease, inactivates members of the mitogen-activated protein kinase family of signaling molecules known to be involved in the responses to stress and inflammation $(23,24)$. In vitro evidence shows that lethal toxin in small doses stimulates macrophages to produce IL- $1 \beta$ and TNF and in large doses causes lysis of macrophages (25). In the process of macrophage death, it has been hypothesized that there is an apparent rapid and overwhelming release of proinflammatory cytokines, which is thought to result in the "sudden death" seen in inhalational anthrax. Thus, lethal toxin is thought to be the principal cause of death in anthrax infection through a macrophagedependent process (26). Protective antigen plays the important role of allowing intracellular entry of the other two factors by binding to them. Although it has no direct toxic effects, strains of bacteria deficient in protective antigen production are far less virulent than the wild-type bacterium $(27,28)$. However, because of their capsule, anthrax bacteria are more difficult to phagocytose and eliminate than typical gram-negative bacteria. Thus, anthrax infection leads to shock accompanied by large bacterial counts systemically.

Mathematical model of anthrax infection-To effect these changes to our previously published model of gram-negative sepsis (13), new equations were added, or existing equations were modified. The model's immune parameters that were fixed using the gramnegative infection scenarios were not modified (see "On-line Supplementary Data"). The above factors and mechanisms were incorporated into a mathematical model that also included elements of acute inflammation and organ damage/dysfunction that are known to accompany anthrax infection. We initially developed a model of acute inflammatory response to a generic gram-negative bacterium (13). This model consists of a system of ordinary differential equations that model the time course of local concentration or cell density of the key components of this response such as pathogen, pathogen-derived toxins, proinflammatory and anti-inflammatory mediators, the coagulation cascade, and global tissue damage/dysfunction (29). The equations are constructed from influence diagrams depicting known biological interactions among model components as documented in the existing scientific literature. We used a previously developed model calibrated on the immune response to endotoxin and trauma/hemorrhage in rodents as starting parameter values (30). Parameters were then adjusted manually to qualitatively reproduce literature data or biologically plausible behavior in the absence of literature data. The level of detail used in the model is aimed at capturing the "design" of the system and at providing a qualitative understanding of the major drivers of the process, rather than quantitative predictions of actual concentrations. 
Predicting the effectiveness of treatment and prevention strategies-One of the most common treatment methods for all infections, including anthrax, is antibiotic administration. Antibiotics may work for cutaneous and gastrointestinal anthrax. However, it has been widely accepted that, by the time the symptoms of inhalational anthrax are present, it is often too late for antibiotic therapy to be effective $(5,31,32)$. Several vaccines have been developed to prevent anthrax outbreaks in animals (33-35). However, data about the efficacy of human vaccines are understandably scarce. Vaccines prepared for use in humans consist of "cell-free" filtrates of the bacilli produced to increase protective antigen content. The vaccine approved by the US Food and Drug Administration is such a preparation, precipitated in alum. There are no controlled clinical trials that explore the efficacy of this vaccine for inhalational anthrax. Below, we describe simulations that may indicate optimal strategies for the use of this vaccine.

To investigate the potential effectiveness of treatment combinations, we simulated the administration of antibiotics at $0,12,24,48,72$, and $96 \mathrm{~h}$ after spore germination with or without a vaccine targeting protective antigen, eliminating $0 \%, 90 \%$, or $100 \%$ of available protective antigen. Lower clearance of protective antigen did not yield results tangibly different from controls. Including controls, 21 different strategies were evaluated. A simulated population of 1,000 cases was created where individuals varied with respect to spore inoculum, bacterial growth rate, and propensity to mount an inflammatory response (early proinflammatory $[\mathrm{Cp}]$ production, late proinflammatory $[\mathrm{CpL}]$ production, antiinflammatory production, and NO production by neutrophils and macrophages). For each individual, model parameters quantifying these processes were drawn from a uniform distribution, with bounds ranging from $50 \%$ to $200 \%$ of the baseline parameter value (see on-line supplementary data at www.shockjournal.com), except for inoculum size where log (inoculum) was uniformly distributed resulting in inoculum ranging over $3 \log$ scales. This cohort was submitted to each treatment arm, and the outcome of the simulation was evaluated as death assessed at 2 weeks (13). Death was defined as cumulative tissue damage exceeding 250 arbitrary units, close to the minimum damage in controls and thus expected to have very high mortality. To evaluate host and pathogen factors most susceptible to predict successful response to combination therapy, we constructed a logistic regression model. The outcome variable was the response to combination therapy (for the purpose of this simulation, we chose to compare the combination of a vaccine fully effective at binding protective antigen and antibiotic intervention at $72 \mathrm{~h}$ compared with a fully effective vaccine alone), and the independent predictor factors included propensity to produce early proinflammatory mediators, late proinflammatory mediators, anti-inflammatory mediators, and NO production as host factors and size of spore inoculum and anthrax growth rate as pathogen factors. The population of 1,000 was divided in quartiles of increasing intensity of the factors (I-IV). Odds ratios for quartiles I to III were generated, using quartile IV as reference category. Goodness-of-fit of the model was evaluated by Hosmer-Lemeshow C statistic and area under the receiver operating characteristic curve $(36,37)$.

\section{RESULTS}

\section{Biosimulation of the clinical course of inhalational anthrax}

In Figures 2 and 3, we show the time course of some of the model components in response to gram-negative bacteria and for which other qualitative simulations have been carried out both using our equation-based models of varying degrees of detail $(13,38-40)$ and using agent-based models (41). The "healthy" and "shock" scenarios are shown in Figures 2 and 3, respectively. In Figure 2, the bacteria grow and immune mediators grow in response. Pathogen is cleared, inflammation resolves as the immune mediators, and tissue damage/ dysfunction return to background values. Such a scenario is interpreted as "healthy." In 
Figure 3, the bacteria grow and trigger inflammation. Although pathogen is cleared, immune mediators and tissue damage/dysfunction remain elevated. We interpret this to be septic shock that could result in multiorgan failure and death (42).

We modified the model to simulate the typical course of anthrax infection by introducing equations describing the specific properties of the anthrax bacterium. We introduce spores that are ingested by resting macrophages. Then, we consider the events after spore germination at $\mathrm{t}=0$. The growing bacteria release protective antigen, lethal factor, and edema factor (but no endotoxin), which combine, as previously described, to form lethal toxin and edema toxin. Lethal toxin is taken up by resting macrophages and, in large quantities, causes cell lysis and massive release of IL-1 (26). Edema toxin increases the population of neutrophils but decreases their capacity to produce superoxide and NO (43), which mediate bacterial killing $(44,45)$. Because of their capsule, anthrax bacteria are more difficult to phagocytose and eliminate than typical gram-negative bacteria. Thus, anthrax infection leads to shock accompanied by large bacterial counts systemically. To effect these changes to the model of gram-negative sepsis, new equations were added, or existing equations were modified. However, the model's immune parameters that were fixed using the gram-negative infection scenarios were not modified (see "On-line Supplementary Data").

We next simulated an immune response induced by inhalation of anthrax spores. As seen in Figure 4, the anthrax bacterium elicits a heightened immune response. Tissue damage/ dysfunction (representative of organ damage and, presumably, lethality) remains elevated for several hours after the onset of germination. Bacteria are not cleared despite high levels of immune mediators. To offer a more transparent comparison between gram-negative infection and anthrax, we kept the number as well as intrinsic growth and death parameters of all bacteria the same. Thus, severe septic shock is the outcome of infection with anthrax bacterium at an inoculum that is nonlethal in the case of gram-negative bacteria.

\section{Predictions from the model}

We simulated therapy consisting of vaccination alone or in combination with antibiotic administration in the setting of an anthrax infection. In our model, an antibiotic was simulated as a chemical that would kill live bacteria on contact. The vaccine was simulated by changing the parameter of production of protective antigen, thereby simulating antibodies that neutralize protective antigen and reduce the availability of protective antigen to form complexes with lethal factor and edema factor.

Vaccine against protective antigen-Our mathematical model suggests that effective vaccination against protective antigen results in tissue damage/dysfunction that is somewhat mitigated (Fig. 5). Vaccination against protective antigen, a key stimulator of the immune response, results in a lowering of inflammation and tissue damage/dysfunction but also in a higher pathogen load. Protective antigen aids in the production of lethal factor and edema factor. Lethal factor is implicated in the systemic release of proinflammatory cytokines. Because protective antigen is cleared from the system by neutralizing antibodies, this intervention results in a systemic anti-inflammatory effect. Although this clearance eliminates the risk of uncontrolled inflammation and ensuing organ failure, the bacterial particles may not be eliminated. Thus, administration of vaccine against protective antigen alone could result in uncontrolled bacterial growth, which could also lead to death (Fig. 5). However, the simulations described above were conducted using a unique set of model parameters corresponding to a very specific genetic disposition. The outcome of an infection depends on several factors, including the virulence of invading pathogen, the size of the inoculum, and the patients' coexisting conditions, age, and genotype, especially as it relates 
to innate immunity (8). Such factors seem to hold true in mouse models as well $(46,47)$. In our model, varying the capacity of the macrophages and neutrophils to produce early cytokines varies the effectiveness of the vaccine. We find that there are reasonable parameter values, in the presence of which, the elimination of protective antigen alone, through the action of a vaccine, is sufficient to restore health (Fig. 6). Hence, we suggest that the strategy of using antiprotective vaccine alone can work for some individuals. When circulating neutrophils respond strongly to the bacterial coat, it effectively counteracts the immune suppression caused by smaller amounts of protective antigen. Increased production of NO aids in clearing bacteria and is generally protective in the setting of bacterial infection $(48,49)$, including anthrax $(50,51)$.

Efficacy of Combination Therapy-We next simulated the effects of different timing and combinations of these therapies. In Figure 7, we show a simulation of the predicted efficacy of pre-existing antibodies to protective antigen in the presence or absence of antibiotics. This simulation is for one set of genetic parameters. In all cases, we show tissue damage/dysfunction where rising tissue damage/dysfunction is equated with death, whereas damage/dysfunction that returns to baseline is synonymous with survival. We first simulated anthrax infection by itself (Fig. 7; case 1) and suggest that this situation would result in death (rising tissue damage/dysfunction). The administration of antibiotics at $\mathrm{t}=0$ (Fig. 7; case 2; an unlikely scenario in an anthrax outbreak) should resolve the infection and result in survival (falling tissue damage/dysfunction). The administration of antibiotics at $\mathrm{t}=48 \mathrm{~h}$ (Fig. 7; case 3; a likely clinical scenario in an anthrax outbreak) is predicted to result in death essentially identical to that of case 1, again in agreement with published results showing a lack of efficacy of late antibiotic administration in anthrax-infected mice (32). The presence of pre-existing circulating antibodies to protective antigen (Fig. 7; case 4; the anthrax vaccine case) is predicted to result in reduced damage/dysfunction as compared with case 1 or 3 , but this effect is not as dramatic as that observed in case 2 . This result is not immediately classifiable as death. However, if a patient has pre-existing circulating antibodies to protective antigen and receives antibiotics at $\mathrm{t}=48 \mathrm{~h}$ (Fig. 7; case 5; a case representing a vaccinated individual that is infected with anthrax), improvement is predicted relative to cases 1,3 , and 4 (but not case 2). Thus, the simulation predicts that a combination of pre-existing circulating antibodies to protective antigen, along with timely administration of antibiotics, is a realistic and effective strategy to deal with sepsis caused by anthrax.

To examine the robustness of those predictions to varying determinants of the host-pathogen response, we carried out simulations of different combinations of times of initiation of antibiotic administration and vaccine efficacy in a cohort of genetically dissimilar individuals inoculated with different number of spores (Table 1). Individuals receiving vaccine without antibiotics showed limited protection. However, we predict that vaccination could provide a very significant window of opportunity for successful antibiotic treatment, up to $96 \mathrm{~h}$ after clinical disease is apparent (Table 1). Finally, we examined host and pathogen factors that predicted a favorable response to combination therapy (fully effective vaccine and antibiotics at $72 \mathrm{~h}$; mortality rate, $26.8 \%$ ), compared with vaccine alone (mortality rate, $87.1 \%$ ). The corresponding logistic regression model fit the data well, with a Hosmer-Lemeshow $\mathrm{C}$ statistic of $11.6(P=0.17)$ and receiver operating characteristic area under the curve of 0.79 . The percentage of correct classification was $75.3 \%$. Exponentiation of the regression coefficients yields the odds of response to combination therapy compared with quartile IV. Low inoculum size and anthrax growth rate resulted in a better response to combination therapy (higher odds ratio; Fig. 8). More surprisingly, high and low early proinflammatory producers had a worse chance of response than those in the middle quartiles, whereas high $\mathrm{CpL}$ producers had the least favorable response. Propensity to NO production had minimal effect on the response to therapy. 


\section{DISCUSSION}

Anthrax is an imminent bioterrorism concern because it is relatively easy to obtain, manipulate, and disperse; has multiple modes of infection; and causes morbidity and mortality rapidly, even without genetic alterations to increase antibiotic resistance (31, 52,

53). Our approach to understanding anthrax infection is to consider inhalational anthrax as a specialized form of septic shock and to model mathematically the response to infection. The mathematical model presented is capable of reproducing many of the features of bacterial sepsis and physiological effects on blood pressure and tissue damage/dysfunction $(13,14)$. This model was modified to simulate features specific to anthrax infection, including the antiphagocytic capsule, the synthesis of edema toxin and lethal toxin from their components, chemoattraction of neutrophils and subsequent suppression of their oxidative burst, and activation and subsequent destruction of macrophages. Anthrax infection simulated with these properties results in septic shock with increased levels of cytokines, damage, and decreased blood pressure and bacterial load that is interpreted as leading to death. At an identical inoculum, a generic gram-negative bacterium without these features elicits recoverable inflammation.

A recent study by Vietri et al. (54) demonstrated that combination therapy consisting of continuous antibiotic administration to rhesus macaque monkeys, starting at 1 to $2 \mathrm{~h}$ after inhalational exposure to anthrax spores, would be ineffective by itself but would potentiate the action of postexposure vaccine. In the present modeling study, we sought to examine the converse question (i.e., Would a vaccinated first responder fare better upon inhalational exposure to anthrax [given a reasonable, but likely delayed, availability of antibiotics]?). As observed in animal experiments, the model suggests that antibiotics administered early in the course of infection are useful in clearing the infection and restoring homeostasis. Late administration of antibiotics is not efficacious because it cannot alter the course of the infection (32). We carried out our analysis further using a simulated cohort of patients varying in their genetic ability to mount an immune response and in the magnitude of the infectious challenge to predict the characteristics of patients likely to benefit from combinations of anthrax vaccine and antibiotics. Our mathematical modeling suggests that vaccination against protective antigen is far from being universally effective in preventing severe disease. Experimental evidence about the efficacy of the anthrax vaccine in humans is sparse. Our model suggests that vaccines, on their own, may be effective in clearing anthrax infection only in some individuals. The presence of protective antigen in the sequence of anthrax infection incites strong proinflammatory responses in the body, predisposing it to tissue damage/dysfunction and eventually septic shock. Therefore, clearance of protective antigen has a systemic anti-inflammatory effect that results, in a generic case, in failure to clear infection. Given favorable circumstances, such as a lowvirulence pathogen (or small inoculum) and a well-calibrated dosage of vaccine, administration of the vaccine alone could restore health. Individuals with strong initial immune response to the bacterial coat and a muted late inflammatory wave may be cured with vaccination alone.

However, in the scenario of depleted protective antigen, our simulations suggest that the main instigator of the immune response is the growing pathogen alone. In these simulations, the chances of an inadequate immune response resulting in persistent infection and subsequent systemic inflammation are high. Late administration of antibiotics actually helps in clearing pathogen and restoring the body to health. Hence, a combination therapy to downregulate inflammation and to later clear bacteria is likely to be more successful for a large population. Likewise, the combination of vaccination (i.e., pre-existing antibodies to protective antigen) coupled with antibiotics given as late as $96 \mathrm{~h}$ after clinical disease is predicted to be more effective than either modality alone. Our simulations of combination 
therapies were analyzed as to suggest characteristics of patients likely to particularly benefit from such combination therapy and predicted host and pathogen factors that would be associated with a favorable response to antibiotic rescue therapy in a manner similar to that carried out previously in the setting of gram-negative sepsis (13). Surprisingly,

proinflammatory production potential is predicted to have a U-shape relationship to response to combination therapy, with the middle two quartiles responding best to salvage therapy. A similar trend is predicted for anti-inflammatory production potential. Nitric oxide production potential is predicted not to impact the response to combination therapy. In agreement with this nonintuitive prediction, and despite studies that suggest a role for iNOS-derived NO in the successful response against anthrax $(55,56)$, Kalns et al. (57) suggested that mice deficient in iNOS are not protected from anthrax infection. Therefore, our simulations points to potential biomarkers to evaluate the potential of a therapeutic response to antibiotics in sick previously vaccinated individuals. We predict that vaccines that are designed to be more efficient in clearing protective antigen are unlikely to be more useful in combating anthrax infection. Instead, future developmental efforts should focus on creating a vaccine that creates a strong antibody response to the bacterial coat and other bacterial components in addition to protective antigen. Such a vaccine may ensure that septic shock is prevented and that anthrax infection is also cleared, with minimal subsequent interventions.

There are limitations to our exploration. We based our mathematical model on the widely accepted hypothesis that lysis of macrophages by lethal toxin and subsequent release of proinflammatory cytokines takes the system into septic shock and death. More recent studies have suggested that anthrax mortality may also be partly because of noninflammatory mechanisms including hypoxia-induced liver failure in which a Fas ligand-dependent process has been implicated. Mice injected with anthrax lethal toxin intraperitoneally demonstrated no evidence of TNF production or symptoms of systemic inflammation (tissue hypoxia, liver necrosis, and pleural edema), although an initial spike of IL- $1 \beta$ concomitant with macrophage lysis was observed (58). However, these observations were based on intraperitoneal injections of anthrax toxins and may therefore be more relevant for the pathogenesis of gastrointestinal rather than inhalational anthrax. Additionally, our acute inflammation model takes into consideration well-vetted mechanisms and does not include many agents whose effects are still being elucidated. The values of the various parameters in our model were derived from-but because of the modifications inherent in modeling, anthrax infection are not identical to- those of previous models published by our group (14). Parameter identification is an ill-posed inverse problem where several sets of parameters could provide equally good fits and preserve biological plausibility when simulations of experimental scenarios outside the calibration scenarios are preserved. We have recently demonstrated the use of an ensemble of models sharing the same framework but with different parameter values-all of which could plausibly explain the inflammatory derangements characteristic of gene-deficient mice (59). In future studies, such ensembles may augment our current methodology for carrying out simulated clinical trials. Despite these limitations, our model duplicates the published features of anthrax infection, both at the cellular and organism levels.

In conclusion, we have simulated the mechanism of anthrax vaccines using a mathematical model based on well-accepted hypotheses about disease pathogenesis. Insights from such simulations should be useful in drawing broad conclusions, especially considering the dearth of experimental evidence. We suggest that our mathematical model, in conjunction with prospective data in a relevant preclinical species, could be used to improve the likelihood of efficacy of anthrax prophylaxis, vaccination, and therapy. 


\section{Supplementary Material}

Refer to Web version on PubMed Central for supplementary material.

\section{Acknowledgments}

This study was supported by the National Institutes of Health (grants nos. R41-AI-52916 and R01-GM-67240).

\section{References}

1. Abramova FA, Grinberg LM, Yampolskaya OV, Walker DH. Pathology of inhalational anthrax in 42 cases from the Sverdlovsk outbreak of 1979. Proc Natl Acad Sci U S A. 1993; 90:2291-2294. [PubMed: 8460135]

2. Read TD, Salzberg SL, Pop M, Shumway M, Umayam L, Jiang L, Holtzapple E, Busch JD, Smith KL, Schupp JM, et al. Comparative genome sequencing for discovery of novel polymorphisms in Bacillus anthracis. Science. 2002; 296:2028-2033. [PubMed: 12004073]

3. An G. Agent-based computer simulation and sirs: building a bridge between basic science and clinical trials. Shock. 2001; 16:266-273. [PubMed: 11580108]

4. Dhawan B, Desikan-Trivedi P, Chaudhry R, Narang P. Bioterrorism: a threat for which we are ill prepared. Natl Med J India. 2001; 14:225-230. [PubMed: 11547531]

5. Shafazand S, Doyle R, Ruoss S, Weinacker A, Raffin TA. Inhalational anthrax: epidemiology, diagnosis, and management. Chest. 1999; 116:1369-1376. [PubMed: 10559102]

6. Meselson M, Guillemin J, Hugh-Jones M, Langmuir A, Popova I, Shelokov A, Yampolskaya O. The Sverdlovsk anthrax outbreak of 1979. Science. 1994; 266:1202-1208. [PubMed: 7973702]

7. Hanna P. Anthrax pathogenesis and host response. 1998; 225:13-35.

8. Hotchkiss RS, Karl IE. The pathophysiology and treatment of sepsis. N Engl J Med. 2003; 348:138150. [PubMed: 12519925]

9. Nass M. Anthrax vaccine. Model of a response to the biologic warfare threat. Infect Dis Clin North Am. 1999; 13:187-208. viii. [PubMed: 10198799]

10. Little SF. Anthrax vaccines: a development update. BioDrugs. 2005; 19:233-245. [PubMed: 16128606]

11. Brey RN. Molecular basis for improved anthrax vaccines. Adv Drug Deliv Rev. 2005; 57:12661292. [PubMed: 15935874]

12. Fellows PF, Linscott MK, Ivins BE, Pitt ML, Rossi CA, Gibbs PH, Friedlander AM. Efficacy of a human anthrax vaccine in guinea pigs, rabbits, and rhesus macaques against challenge by Bacillus anthracis isolates of diverse geographical origin. Vaccine. 2001; 19:3241-3247. [PubMed: 11312020]

13. Clermont G, Bartels J, Kumar R, Constantine G, Vodovotz Y, Chow C. In silico design of clinical trials: a method coming of age. Crit Care Med. 2004; 32:2061-2070. [PubMed: 15483415]

14. Chow CC, Clermont G, Kumar R, Lagoa C, Tawadrous Z, Gallo D, Betten B, Bartels J, Constantine G, Fink MP, et al. The acute inflammatory response in diverse shock states. Shock. 2005; 24:74-84. [PubMed: 15988324]

15. Janeway CA Jr, Medzhitov R. Innate immune recognition. Annu Rev Immunol. 2002; 20:197-216. [PubMed: 11861602]

16. Jaeschke H, Smith CW. Mechanisms of neutrophil-induced parenchymal cell injury. J Leukoc Biol. 1997; 61:647-653. [PubMed: 9201255]

17. Levy MM, Fink MP, Marshall JC, Abraham E, Angus D, Cook D, Cohen J, Opal SM, Vincent JL, Ramsay G. 2001 SCCM/ESICM/ACCP/ATS/SIS International Sepsis Definitions Conference. Crit Care Med. 2003; 31:1250-1256. [PubMed: 12682500]

18. Pinsky MR, Vincent JL, Deviere J, Alegre M, Kahn RJ, Dupont E. Serum cytokine levels in human septic shock. Relation to multiple-system organ failure and mortality. Chest. 1993; 103:565-575. [PubMed: 8432155] 
19. Read TD, Salzberg SL, Pop M, Shumway M, Umayam L, Jiang L, Holtzapple E, Busch JD, Smith KL, Schupp JM, et al. Comparative genome sequencing for discovery of novel polymorphisms in Bacillus anthracis. Science. 2002; 296:2028-2033. [PubMed: 12004073]

20. Abramova FA, Grinberg LM, Yampolskaya OV, Walker DH. Pathology of inhalational anthrax in 42 cases from the Sverdlovsk outbreak of 1979. Proc Natl Acad Sci U S A. 1993; 90:2291-2294. [PubMed: 8460135]

21. Dai Z, Koehler TM. Regulation of anthrax toxin activator gene (atxA) expression in Bacillus anthracis: temperature, not CO2/bicarbonate, affects AtxA synthesis. Infect Immun. 1997; 65:2576-2582. [PubMed: 9199422]

22. O’Brien J, Friedlander A, Dreier T, Ezzell J, Leppla S. Effects of anthrax toxin components on human neutrophils. Infect Immun. 1985; 47:306-310. [PubMed: 3917427]

23. Bonventre JV. Molecular response to cytotoxic injury: role of inflammation, MAP kinases, and endoplasmic reticulum stress response. Semin Nephrol. 2003; 23:439-448. [PubMed: 13680533]

24. Kumar S, Boehm J, Lee JC. p38 MAP kinases: key signalling molecules as therapeutic targets for inflammatory diseases. Nat Rev Drug Discov. 2003; 2:717-726. [PubMed: 12951578]

25. Friedlander AM. Macrophages are sensitive to anthrax lethal toxin through an acid-dependent process. J Biol Chem. 1986; 261:7123-7126. [PubMed: 3711080]

26. Hanna PC, Acosta D, Collier RJ. On the role of macrophages in anthrax. Proc Natl Acad Sci U S A. 1993; 90:10198-10201. [PubMed: 8234277]

27. Brossier F, Weber-Levy M, Mock M, Sirard JC. Protective antigen-mediated antibody response against a heterologous protein produced in vivo by Bacillus anthracis. Infect Immun. 2000; 68:5731-5734. [PubMed: 10992478]

28. Pezard C, Weber M, Sirard JC, Berche P, Mock M. Protective immunity induced by Bacillus anthracis toxin-deficient strains. Infect Immun. 1995; 63:1369-1372. [PubMed: 7890396]

29. Clermont, G.; Chow, CC.; Constantine, GM.; Vodovotz, Y.; Bartels, J. Classification, Clustering, and Data Mining Applications. Proceedings of the IFCS. New York: Springer; 2004. Mathematical and statistical modeling of acute inflammation; p. 457-467.

30. Chow CC, Clermont G, Kumar R, Lagoa C, Tawadrous Z, Gallo D, Betten B, Bartels J, Constantine G, Fink MP, et al. The acute inflammatory response in diverse shock states. Shock. 2005; 24:74-84. [PubMed: 15988324]

31. Dixon TC, Meselson M, Guillemin J, Hanna PC. Anthrax. N Engl J Med. 1999; 341:815-826. [PubMed: 10477781]

32. Kalns J, Morris J, Eggers J, Kiel J. Delayed treatment with doxycycline has limited effect on anthrax infection in BLK57/B6 mice. Biochem Biophys Res Commun. 2002; 297:506-509. [PubMed: 12270123]

33. Friedlander AM, Welkos SL, Ivins BE. Anthrax vaccines. Curr Top Microbiol Immunol. 2002; 271:33-60. [PubMed: 12224523]

34. Greenfield RA, Bronze MS. Prevention and treatment of bacterial diseases caused by bacterial bioterrorism threat agents. Drug Discov Today. 2003; 8:881-888. [PubMed: 14554016]

35. Leppla SH, Robbins JB, Schneerson R, Shiloach J. Development of an improved vaccine for anthrax. J Clin Invest. 2002; 110:141-144. [PubMed: 12122102]

36. Lemeshow S, Hosmer DW Jr. A review of goodness of fit statistics for use in the development of logistic regression models. Am J Epidemiol. 1982; 115:92-106. [PubMed: 7055134]

37. Hanley JA. Receiver operating characteristic (ROC) methodology: the state of the art. Crit Rev Diagn Imaging. 1989; 29:307-335. [PubMed: 2667567]

38. Kumar R, Clermont G, Vodovotz Y, Chow CC. The dynamics of acute inflammation. J Theor Biol. 2004; 230:145-155. [PubMed: 15321710]

39. Reynolds A, Rubin J, Clermont G, Day J, Vodovotz Y, Ermentrout GB. A reduced mathematical model of the acute inflammatory response: I. Derivation of model and analysis of antiinflammation. J Theor Biol. 2006; 242:220-236. [PubMed: 16584750]

40. Day J, Rubin J, Vodovotz Y, Chow CC, Reynolds A, Clermont G. A reduced mathematical model of the acute inflammatory response: II. Capturing scenarios of repeated endotoxin administration. J Theor Biol. 2006; 242:237-256. [PubMed: 16616206] 
41. An G. Agent-based computer simulation and sirs: building a bridge between basic science and clinical trials. Shock. 2001; 16:266-273. [PubMed: 11580108]

42. Kumar R, Clermont G, Vodovotz Y, Chow CC. The dynamics of acute inflammation. J Theor Biol. 2004; 230:145-155. [PubMed: 15321710]

43. O’Brien J, Friedlander A, Dreier T, Ezzell J, Leppla S. Effects of anthrax toxin components on human neutrophils. Infect Immun. 1985; 47:306-310. [PubMed: 3917427]

44. Kudoh S, Suzuki K, Yamada M, Liu Q, Nakaji S, Sugawara K. Contribution of nitric oxide synthase to human neutrophil chemiluminescence. Luminescence. 1999; 14:335-339. [PubMed: 10602304]

45. Pacelli R, Wink DA, Cook JA, Krishna MC, DeGraff W, Friedman N, Tsokos M, Samuni A, Mitchell JB. Nitric oxide potentiates hydrogen peroxide-induced killing of Escherichia coli. J Exp Med. 1995; 182:1469-1479. [PubMed: 7595217]

46. McAllister RD, Singh Y, du Bois WD, Potter M, Boehm T, Meeker ND, Fillmore PD, Anderson LM, Poynter ME, Teuscher C. Susceptibility to anthrax lethal toxin is controlled by three linked quantitative trait loci. Am J Pathol. 2003; 163:1735-1741. [PubMed: 14578173]

47. Moayeri M, Haines D, Young HA, Leppla SH. Bacillus anthracis lethal toxin induces TNF-alphaindependent hypoxia-mediated toxicity in mice. J Clin Invest. 2003; 112:670-682. [PubMed: 12952916]

48. Cobb JP, Hotchkiss RS, Swanson PE, Chang K, Qiu Y, Laubach VE, Karl IE, Buchman TG. Inducible nitric oxide synthase (iNOS) gene deficiency increases the mortality of sepsis in mice. Surgery. 1999; 126:438-442. [PubMed: 10455918]

49. Nathan CF, Hibbs JB Jr. Role of nitric oxide synthesis in macrophage antimicrobial activity. Curr Opin Immunol. 1991; 3:65-70. [PubMed: 1711326]

50. Moayeri M, Martinez NW, Wiggins J, Young HA, Leppla SH. Mouse susceptibility to anthrax lethal toxin is influenced by genetic factors in addition to those controlling macrophage sensitivity. Infect Immun. 2004; 72:4439-4447. [PubMed: 15271901]

51. Raines KW, Kang TJ, Hibbs S, Cao GL, Weaver J, Tsai P, Baillie L, Cross AS, Rosen GM. Importance of nitric oxide synthase in the control of infection by Bacillus anthracis. Infect Immun. 2006; 74:2268-2276. [PubMed: 16552057]

52. Inglesby TV, Henderson DA, Bartlett JG, Ascher MS, Eitzen E, Friedlander AM, Hauer J, McDade J, Osterholm MT, O'Toole T, et al. Anthrax as a biological weapon: medical and public health management. Working Group on Civilian Biodefense. JAMA. 1999; 281:1735-1745. [PubMed: 10328075]

53. Meselson M, Guillemin J, Hugh-Jones M, Langmuir A, Popova I, Shelokov A, Yampolskaya O. The Sverdlovsk anthrax outbreak of 1979. Science. 1994; 266:1202-1208. [PubMed: 7973702]

54. Vietri NJ, Purcell BK, Lawler JV, Leffel EK, Rico P, Gamble CS, Twenhafel NA, Ivins BE, Heine HS, Sheeler R, et al. Short-course postexposure antibiotic prophylaxis combined with vaccination protects against experimental inhalational anthrax. Proc Natl Acad Sci U S A. 2006; 103:78137816. [PubMed: 16672361]

55. Moayeri M, Martinez NW, Wiggins J, Young HA, Leppla SH. Mouse susceptibility to anthrax lethal toxin is influenced by genetic factors in addition to those controlling macrophage sensitivity. Infect Immun. 2004; 72:4439-4447. [PubMed: 15271901]

56. Raines KW, Kang TJ, Hibbs S, Cao GL, Weaver J, Tsai P, Baillie L, Cross AS, Rosen GM. Importance of nitric oxide synthase in the control of infection by Bacillus anthracis. Infect Immun. 2006; 74:2268-2276. [PubMed: 16552057]

57. Kalns J, Scruggs J, Millenbaugh N, Vivekananda J, Shealy D, Eggers J, Kiel J. TNF receptor 1, IL-1 receptor, and iNOS genetic knockout mice are not protected from anthrax infection. Biochem Biophys Res Commun. 2002; 292:41-44. [PubMed: 11890668]

58. Moayeri M, Haines D, Young HA, Leppla SH. Bacillus anthracis lethal toxin induces TNF-alphaindependent hypoxia-mediated toxicity in mice. J Clin Invest. 2003; 112:670-682. [PubMed: 12952916]

59. Prince JM, Levy RM, Bartels J, Baratt A, Kane JM 3rd, Lagoa C, Rubin J, Day J, Wei J, Fink MP, et al. In silico and in vivo approach to elucidate the inflammatory complexity of CD14-deficient mice. Mol Med. 2006; 12:88-96. [PubMed: 16953560] 


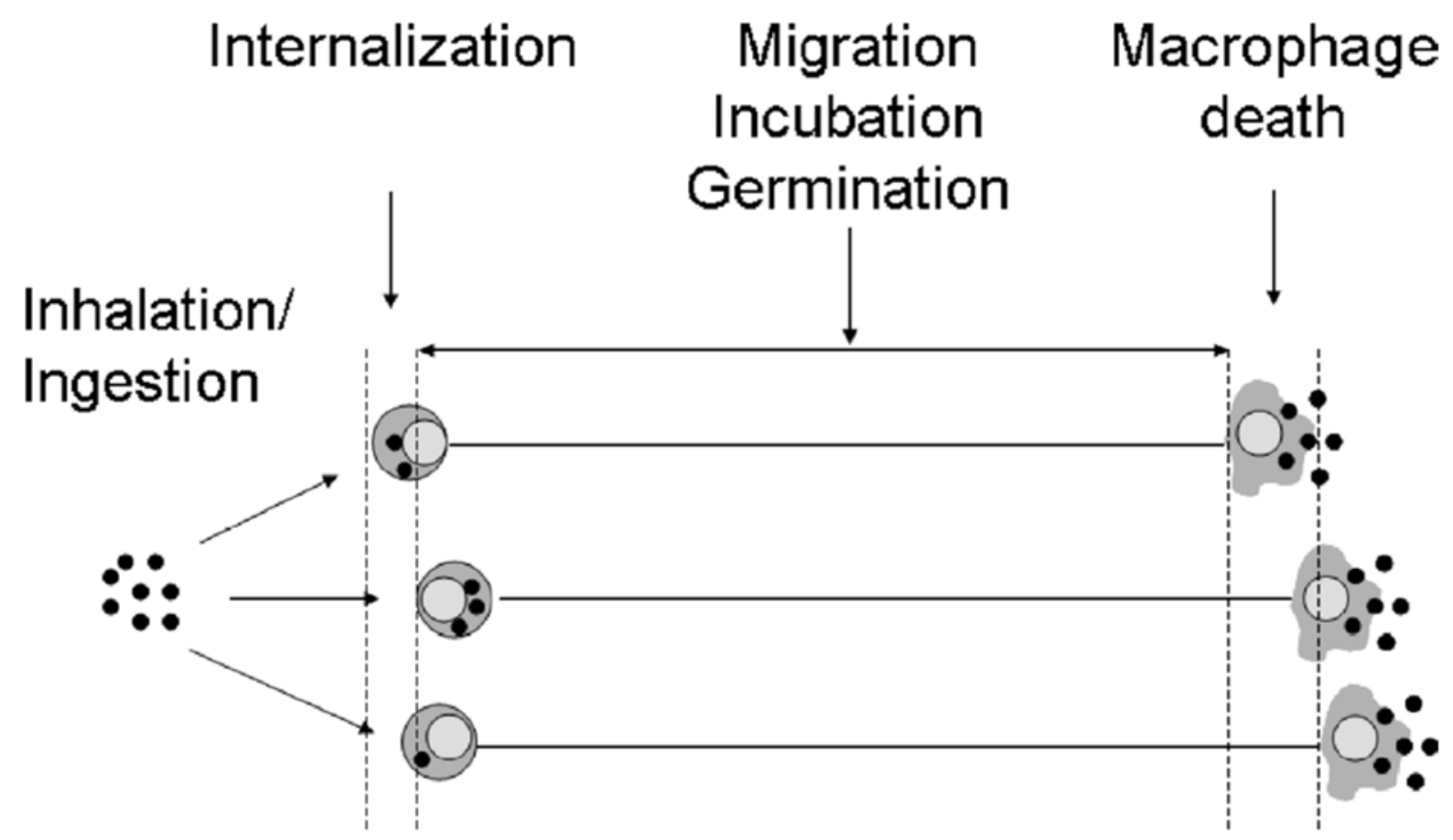

Fig. 1. Schematic of the course of anthrax infection

Infection is initiated by inhalation or ingestion of anthrax spores, which are internalized by monocytes/macrophages/dendritic cells. The spores incubate, and therefore, monocytes and immature dendritic cells spread latent anthrax infection throughout the body. Given appropriate environmental conditions, the spores germinate. 

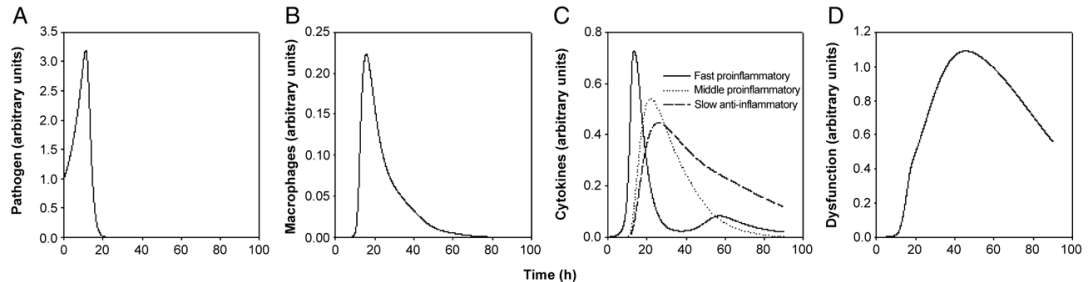

Fig. 2. Simulation of a healthy subject in response to gram-negative bacterial infection A virtual patient is infected by a low dose of gram-negative bacteria (A), which leads to activation of macrophages (B). The macrophages elaborate cytokines (C), including rapidly produced proinflammatory cytokines (Cp; e.g., IL-1 and TNF), more slowly produced proinflammatory cytokines (CpL; e.g., IL-6), and anti-inflammatory cytokines (Ca; e.g., TGF- $\beta 1$ ). Tissue damage/dysfunction (D) rises because of the actions of proinflammatory cytokines and effectors but then decreases because of the actions of anti-inflammatory cytokines. Thus, homeostasis is restored after pathogens are cleared. 

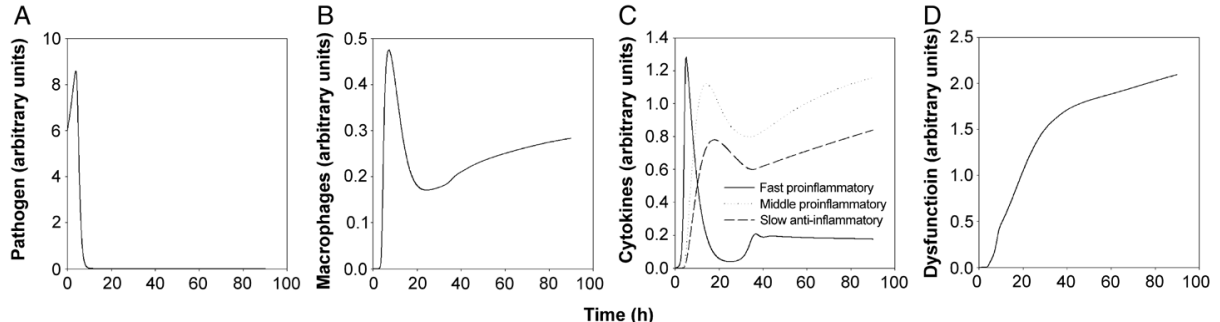

Fig. 3. Simulation of persistent gram-negative sepsis

A virtual patient is infected by a high dose of gram-negative bacteria (A), which leads to activation of macrophages (B). The macrophages elaborate cytokines (C), including rapidly produced (early) proinflammatory cytokines (Cp; e.g., IL-1 and TNF), more slowly (late) produced proinflammatory cytokines (CpL; e.g., IL-6), and anti-inflammatory cytokines (Ca; e.g., TGF- $\beta 1$ ). Tissue damage/dysfunction (D) rises because of the actions of proinflammatory cytokines and effectors and, unlike the case in Figure 1, is not reduced. Thus, the inflammatory response is sustained, with ensuing adverse outcome. 

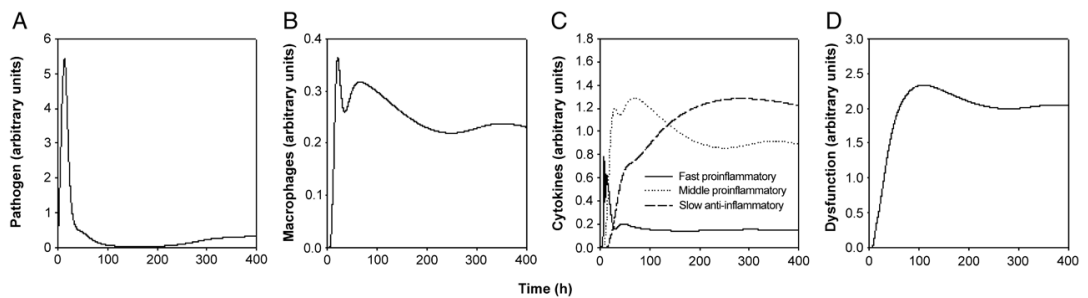

Fig. 4. Modification of the mathematical model of sepsis to account for anthrax-specific effects Comparison of the predicted effect on immune mediators and tissue damage/dysfunction in anthrax infection. Despite an exuberant immune response, the bacterial load and tissue damage/dysfunction remain high (the bacterial growth parameters are the same as that of the gram-negative infection in Fig. 1). 


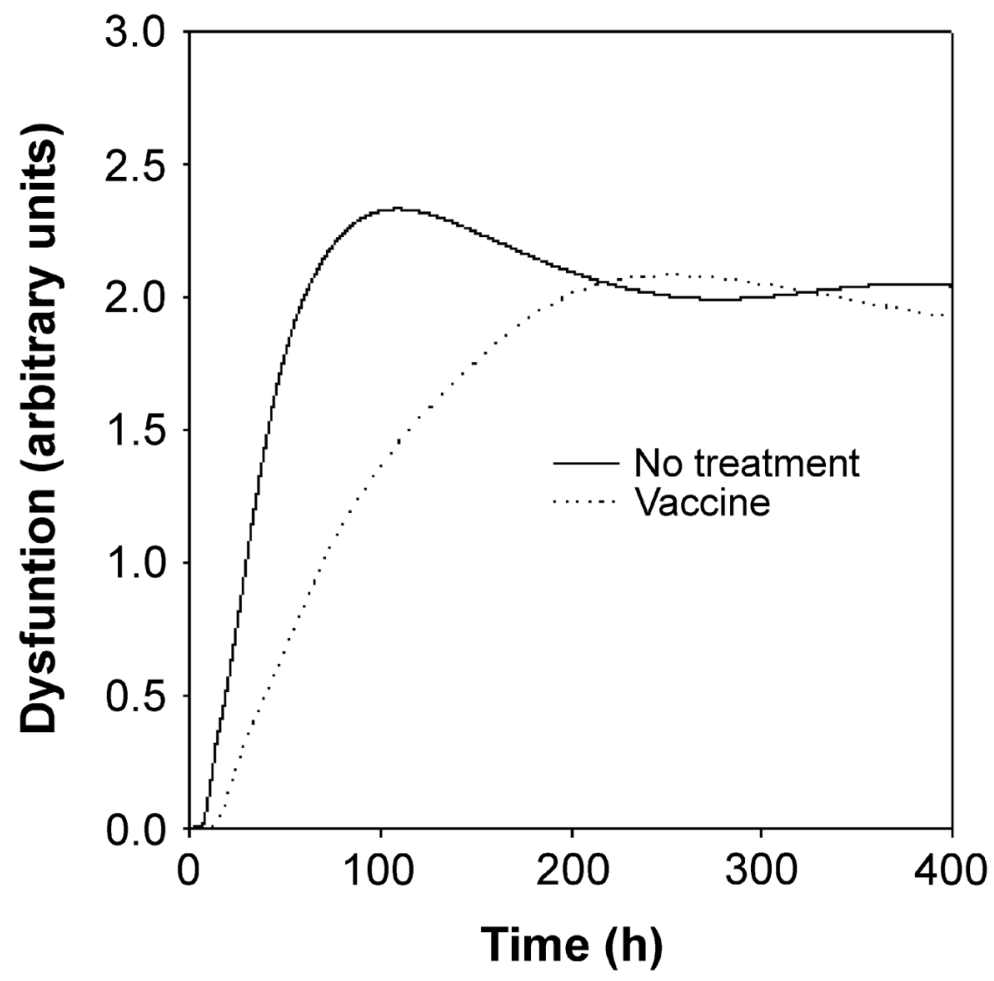

Fig. 5. Vaccination against protective antigen leads to a decrease in the stimulation of the immune response

Vaccination leads to depletion of protective antigen. The primary stimulants are now elements of the bacterial coat, and the response is insufficient to clear bacteria. Therefore, although a downregulated immune response leads to lower damage, it also results in unbounded growth of bacteria. 


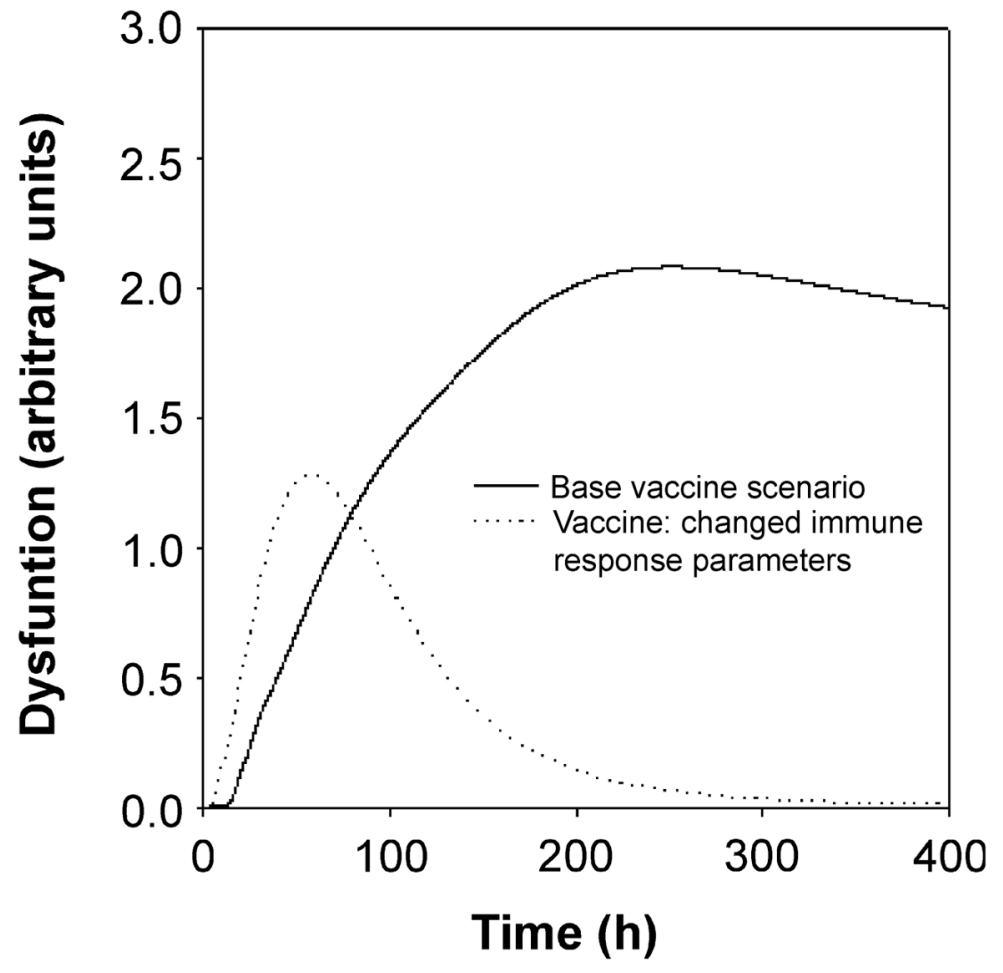

Fig. 6. Simulation with a set of parameters where vaccination alone is sufficient in curing anthrax

Varying the parameters in the model could resemble the inherent variations in a population. In the above simulation, pathogen and vaccine properties were kept the same, but immune response parameters were changed such that neutrophil activation after exposure to pathogen was 8 times the base rate, and NO production by neutrophils was 6 times the base rate for comparable stimuli. Tissue damage/dysfunction, in the presence of antiprotective antigen vaccine, in the case of the base parameter values is compared with the modified set. 


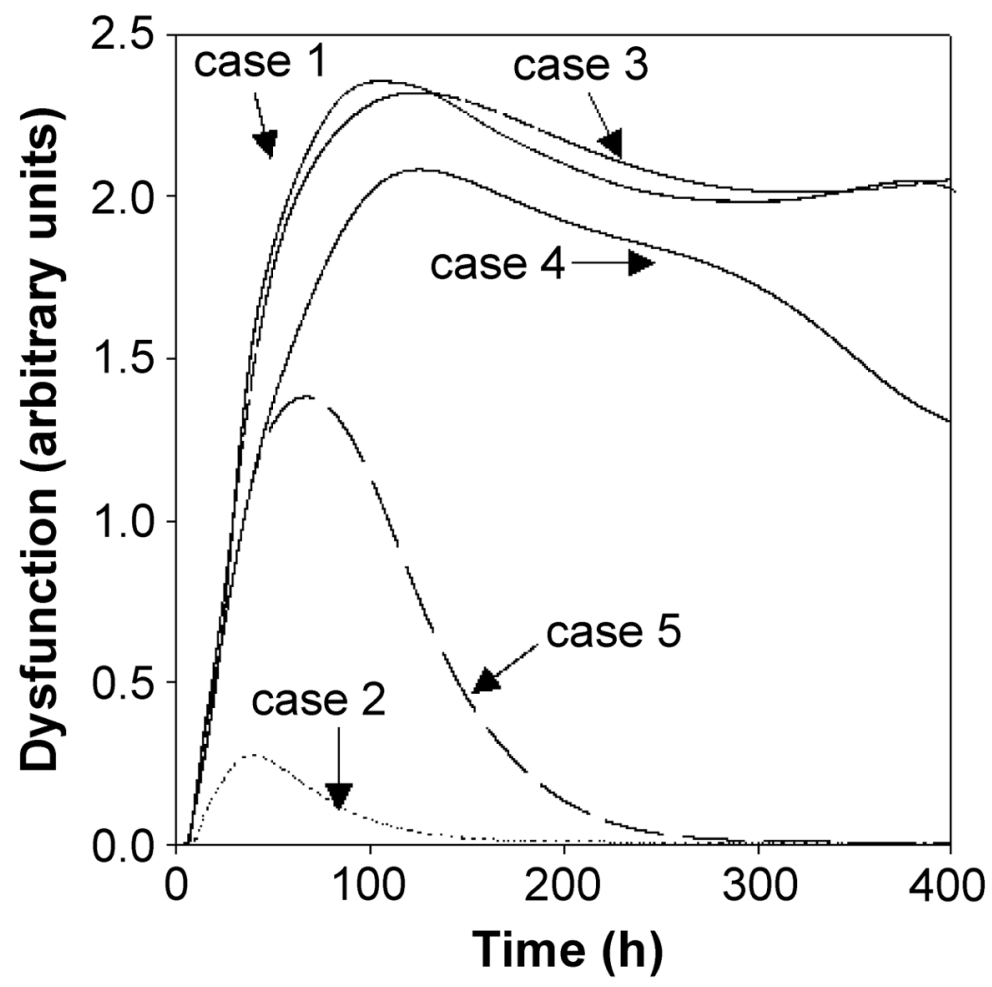

Fig. 7. Simulation of the efficacy of antibiotics and vaccination in inhalational anthrax Simulation of tissue damage/dysfunction under the various circumstances. Case 1, anthrax infection, no antibiotics; case 2, anthrax infection, antibiotic administration at $\mathrm{t}=0$; case 3 , anthrax infection, antibiotic administration at $\mathrm{t}=48$; case 4 , anthrax infection in the setting of vaccination leading to pre-existing circulating antibodies to protective antigen; case 5 , anthrax infection in the presence of vaccination and antibiotic administration at $\mathrm{t}=48 \mathrm{~h}$. 


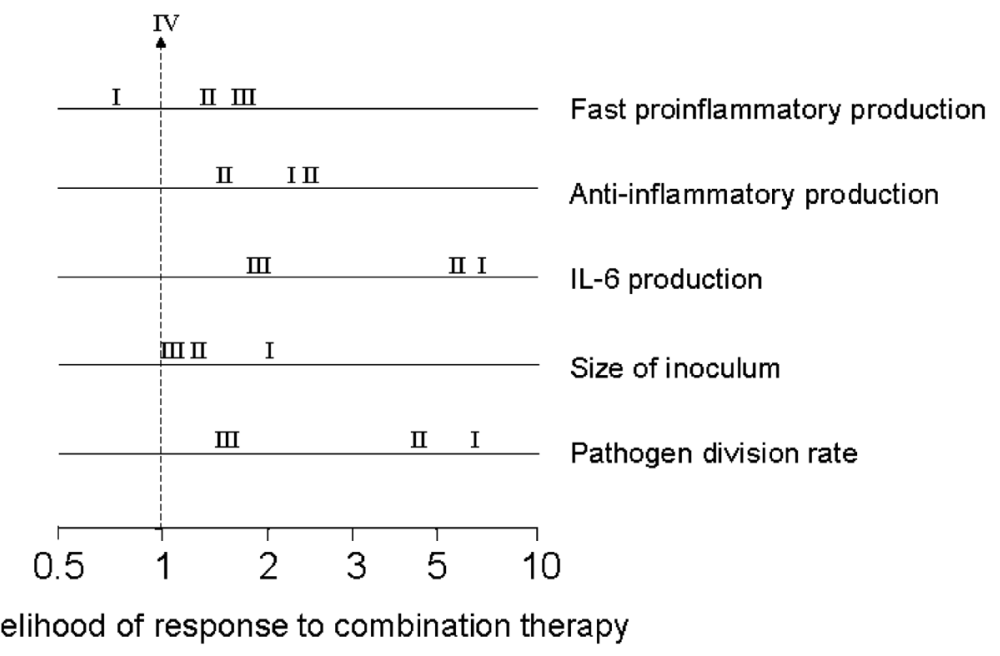

Fig. 8. Predictors of response to combination therapy

A logistic regression model was constructed to determine if variation in host and pathogen factors would impact the response to combination therapy (fully effective vaccine with rescue antibiotics $72 \mathrm{~h}$ after the start of clinical infection, compared with vaccine alone; overall rate of response of $60.3 \%$, reducing mortality from $87.1 \%$ to $26.8 \%$ ). Predictors were ranked by quartiles of increasing value (I-IV), with the highest quartile reflecting the highest inoculum size, pathogen growth rate and propensity to mount a proinflammatory or anti-inflammatory response or produce NO. For each predictor, odds ratios associated with quartiles I to III, using quartile IV as reference (odds ratio, 1), are depicted. Note the nonmonotonic relationship between propensity to mount a short proinflammatory response $(\mathrm{Cp})$ and the response to combination therapy. A similar less marked "dose-response" relationship is seen for anti-inflammatory production. Propensity to NO production did not significantly impact response to combination therapy. 


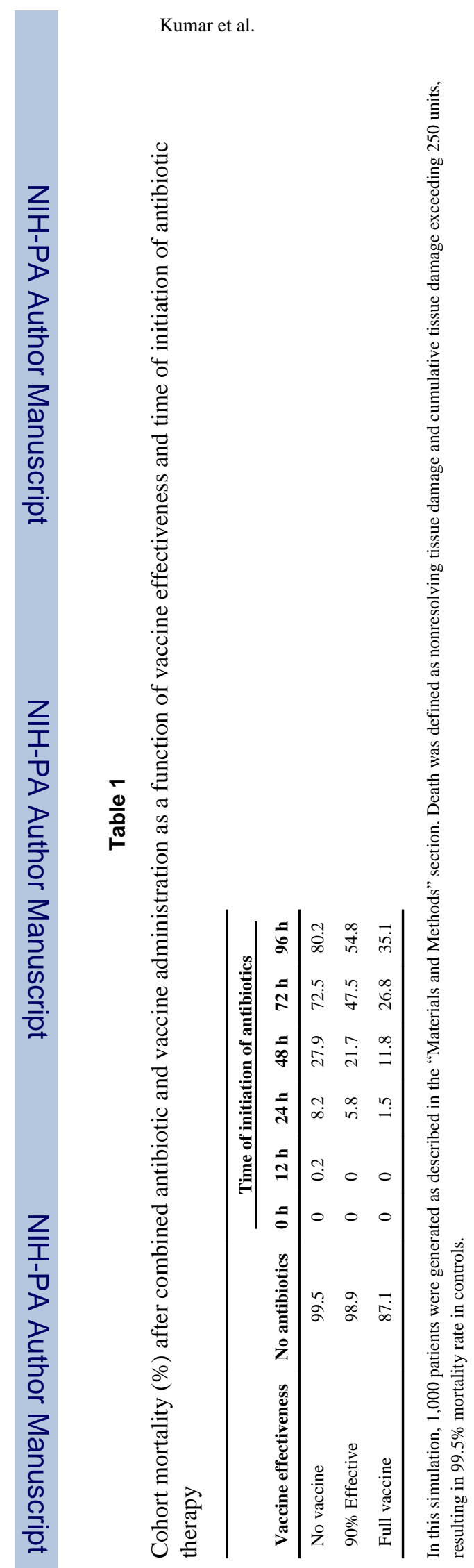

Shock. Author manuscript; available in PMC 2013 May 15. 